\title{
XXIX.
}

\section{Antiquarische Randbemerkungen.}

1. Ueber einige antike Schlaginstrumente.

Unter die guten französischen Bücher, die bei uns zu wenig benutzt werden, zählt der Dictionnaire des antiquités von Daremberg und Saglio. Wir haben ihm vorläufig kaum etwas an die Seite zu stellen. Die kleinen Bemerkungen, die ich im Folgenden vorlege, sind beim Durchblättern der letzten Lieferungen entstanden ${ }^{1}$ ) und sollen ihren Ursprung nicht verleugnen.

II 280 läßt Saglio einen Bron c e-Disk u s aus dem Museum zu Neapel abbilden, der zum Aufhängen bestimmt ist; neben ihm, auf der Breitseite hängt ein Klöppel an einer feinen Kette. Zur Erklärung führt S. zwei Stellen an, aus denen hervorgeht, daß man in der That mit einem Diskus Signale zu geben pflegte. 1) Cicero de Orat. II 5, $21^{2}$ ) auditores [philosophorum] discum audire, quam philosophum malunt $\left.{ }^{3}\right)$ : qui simul ut increpuit, in media oratione... philosophum omnes unctionis causa relinquunt. Wir sind in einem griechischen Gymnasium: der Diskusklang verkündet den Anfang der Leibesübungen. 2) Mare Aurel an Fronto, III 6 p. 70 Naber: discus crepuit, id est pater meus in

1) Daremberg et Saglio, dictionnaire des antiquités, fasc. XIII sqq. $1889-1892$.

2) Saglio citiert falsch Cic. Or. V 2.

3) Natürlich ist das kein Sprichwort, wie nach altem Vorgange wieder Klotz angenommen hat. Vgl. aber die S. $518^{8}$ nachgewiesene Anekdote. 
balneum transisse nuntiatus est. Daraus folgt freilich noch nicht, daB von dem üblichen signal du bain die Rede ist; wir sind in der Umgebung des Kaisers, dessen Ankunft das Signal wohl verkünden soll.

Mit diesen Zeugnissen ist ein drittes zu combinieren, das Saglio übersehen hat. Didymus Schol. Plat. Phaed. p. 381 B.

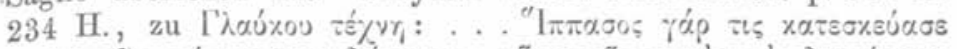

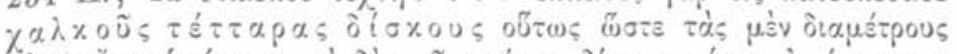

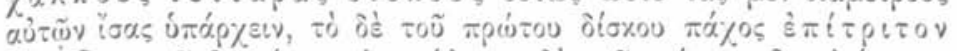

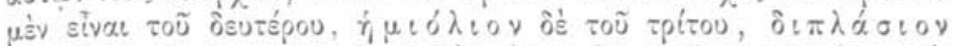

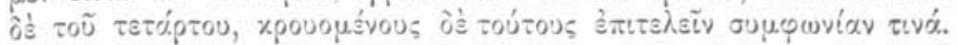

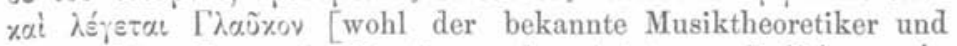

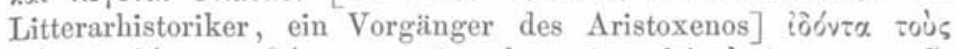

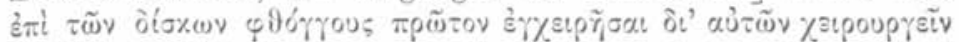
[d. h. zu spielen, vgl. Aristot. Pol. VIII 6, 7 p. 228, 30, Luc.

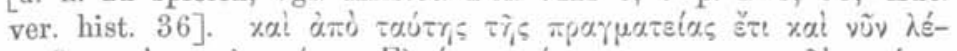

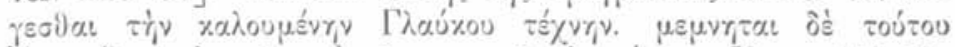

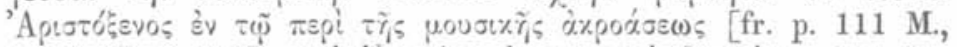

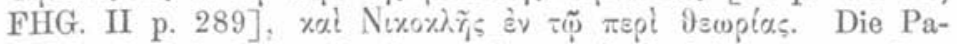
rallelartikel bei den Lexikographen und Sprichwörtersammlern bieten nichts neues (vgl. Jungblut, Quaest. de paroemiographis p. 40); Zenob. volg. II 91 sagt nur deutlicher xai xpovouévous

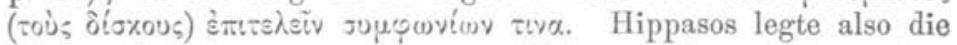
Klangverhältnisse von vier verschieden starken Disken fest, Glaukos bildete die Kunst aus, sie mit einem Schlagstabe zu spielen.

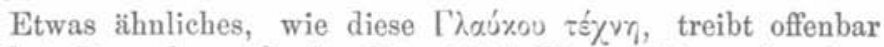
die bei Daremberg - Saglio Fig. 2594 II p. 449 nach einer durchaus antik empfundenen Miniatur abgebildete Dame; sie steht mit zwei Schlagstäben vor einem Brett mit vier verschieden starken Becken; das erste rechter Hand hat sie eben mit dem Stabe berïhrt. Daß die Zahl der Becken zu der Aristoxenosnotiz stimmt, ist bemerkenswerth genug.

Saglio behandelt das Bildehen unter dem Stichwort E c h ei on. Er hätte viel eher an die acetabula erinnern sollen, von denen I 23 in freilich nicht ganz ausreichender Weise gehandelt wird ${ }^{4}$ ). Da auch ein so tüchtiger Kenner der alten Organik, wie C. v. Jan, das Problem m. E. nicht ganz richtig angefaßt hat (Baumeisters Denkm. III 1662 f.), wird sich's lohnen, gründlicher darauf einzugehen. Bei Suidas folgt auf den Hesychischen $\beta$ íos

4) Saglio führt nur die sekundäre Stelle des Isidor (= Cassiod.)

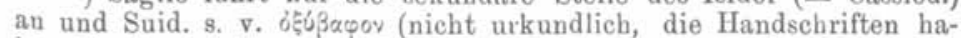
ben die Nachricht $s$. $\Delta\left(0 \times \lambda \tilde{r}_{i} \zeta\right)$, auch meint er, man babe das Instrument contre une autre cymbale ou au moyen d'une baguette geschlagen. Am besten spricht über die Frage Bellermann zum Anonymus $\S 17$ p. 28. 


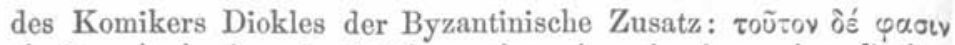

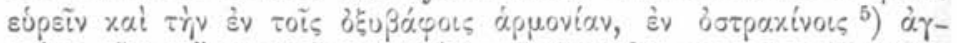

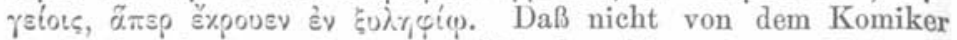
die Rede sein kann, hat schon Meineke (hist. erit. p. 251) ge-

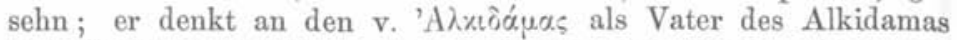
und Musikschriftsteller genannt Diokles von Elaia. Nun gab

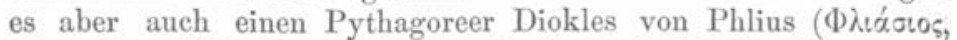
Diog. La. VIII 45, Iamblich. vit. Pyth. § 218). Diesen erwähnte derselbe Aristoxenos, dem wir die Nachricht über das musikalische Experiment des Glaukos verdanken (a. O. $=$ fr. 11. 12 FHG. II p. 273 M.) ; und da in der Suidas-vita als Heimath des Diokles Athen oder Phlius (Airvaios $\ddot{\gamma}_{i}$ (j)íaros) angegeben ist, so ist es meines Erachtens zweifellos, daß die Worte $\ddot{\gamma}_{i}$ (itácos ein Zusatz aus derselben Quelle sind, aus dem die Schlußnotiz geschöpft ist, daß also der Musiker Diokles kein andrer ist, als der bei Aristoxenos erwähnte Pythagoreer

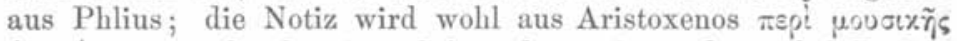

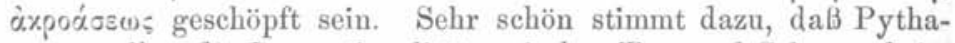
goras selbst die Proportionalität zwischen Ton und Schwere beim Klange verschiedener Schmiedhämmer beobachtet haben soll, s.

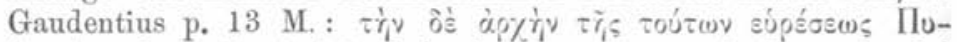

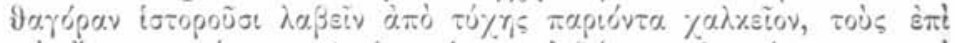

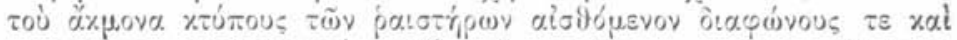

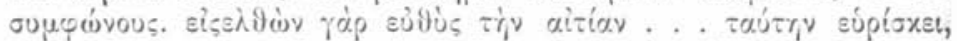

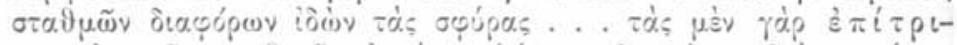

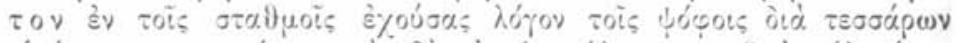

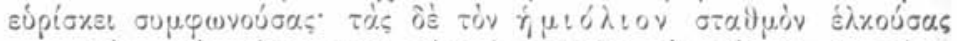

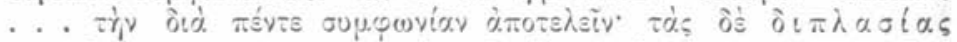

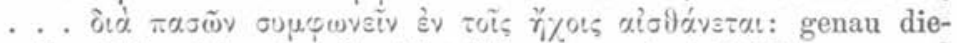
selbe Reihenfolge der Gewichtsdifferenz, wie bei Hippasos und Glaukos. Die Quelle wird wiederum Aristoxenos sein. Denn diese Uberlieferungen von Pythagoras, Diokles, Glankos-Hippasos reichen sich wechselseitig die Hand. Der Meister hat den genialen Einfall; die Schüler bilden ihn weiter und führen ihn in die Praxis ein.

Nun erscheint das Wort acetabulum, dem griechischen b̧úPạpov genau entsprechend, bei Cassiodor de mus. p. 573 ed. 1656 in einem System der Organik, wo an erster Stelle die percussionalia (instrumenta) erklärt werden als acitabula aenea vel argentea vel alia, quae et aliquo rigore percussa reddunt cum suavitate tinnitum. Das Wort taucht auch in den Varia auf, IV 51 p. 163 , an einer sehr schulmäßig gehaltenen Stelle über die verschiedenen im Theater der antiqui gebotenen Kunstgenüsse:

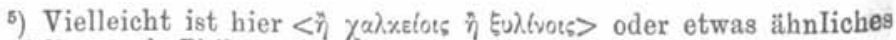
ausgefallen, vgl. Philoponos bei Suid. s. v. áppovi $\alpha$ I p. 747 B. 
Quid acitabulom tinnitus, quid dulcissimi soni referam varia percussione modulamen. v. Jan will überraschender Weise a. O. S. 1663 diese acetabula nicht zu den einzeln gespielten Oxybapha rechnen, sondern zu den paarweise geschlagenen Cymbeln. Das erweist sich aber als durchaus unberechtigt, sobald man den Zusammenhang der Zeugnisse überblickt. Cassiodor entlehnt den Abschnitt de musica, wie er am Anfang und am SchluB (p. 572 und 576) betont, aus einer lateinischen Uebersetzung des oben (S. 516) citierten Aristoxenikers Gaudentius, aus dem Gaudentius Mutiani latinus. Auch in den Varia ist der Ausdruck nur gelehrte Reminiscenz. Wir haben also einfach eine Uebersetzung des griechischen $6 \xi \hat{\beta} \beta$ rọ $\mathrm{zu}$ erkennen. Es ist dieselbe Glas-, Holz- oder Metallharmonika gemeint, wie in den griechischen Quellen. Das bestätigt ja auch bei einiger Ueberlegung der Ausdruck aliquo rigore und varia percussione modulamen, der auf Cymbeln nicht passen würde. Uebrigens mag Cassiodorius solche Instrumente immerhin gehört haben. Auch Philoponos zu Aristot. de an. I (= Suid. s. v. apuovi $\alpha$ I p. 747 B ) scheint sie

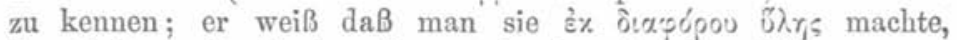

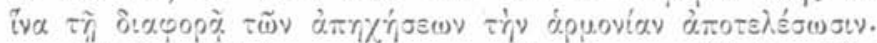

Doch zurück zu der von Saglio angeführten Miniatur.

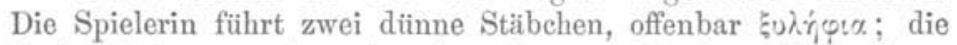
Becher haben geradezu das Aussehn von Näpfen. Das stimmt so gut zu dem wunderlichen Namen und der Beschreibung bei Suidas, daß man mit Zuversicht sagen kann: hier ist eine Oxybapha-Spielerin abgebildet.

Aehnliehe Schlaginstrumente kannten auch die Stifter des berïhmten Weihgeschenkes zu Dodona, auf das man das Sprich-

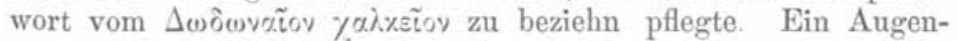
zenge, Polemo, beschreibt es bei Steph. Byz. s. v. $\Delta \omega \hat{o} \omega \dot{v \eta}$ (vermittelt durch den Paroemiographen Aristides) und bei Strabo VII p. 329 sehr anschaulich. Strabo berichtet: $\chi \alpha \lambda \times \varepsilon i o \nu \tilde{\eta}^{2}$

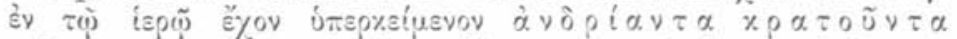

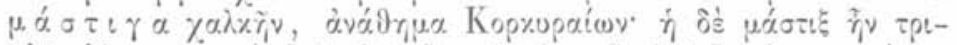

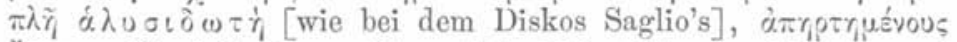

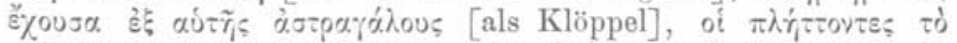

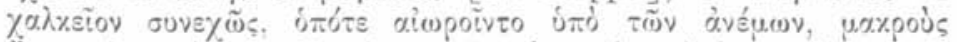

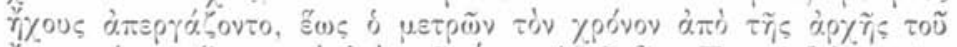

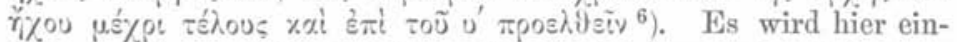
fach der Apparat beschrieben, den Saglio besprochen hat, nur in kunstvollerer Gestaltung. Der àvôpı́. selbst schlägt die

$\left.{ }^{6}\right)$ Die Fassung bei Strabo halte ich für die beste; bei Stephanus und Zenobius tritt Aristides als trübendes Mittel dazwischen (FHG. IV p. 326). Vgl. Preller, Polem. p. 56. Müller FHG. III p. 124. Die Ueberlieferung der Paroemiographen ist spärlich gegenüber der bei Stephanus, vgl. Warnkross, De paroemiographis p. 36 sq. 
Erzscheibe ( $\chi \alpha \lambda x \varepsilon i o v)$, ganz wie unser Kunstgewerbe Tischglocken und Gongs mit Figuren bildet. Auch der Zweck der Vorrichtung wird ursprünglich derselbe gewesen sein, wie in den angeführten Fällen. Daß sie als Aeolslarfe dient. was O. Müller (kl. Schr. I 587) so poesievoll ausgeführt hat ${ }^{7}$ ), macht ganz den Eindruck des Zufälligen und Sekundären. Am ersten wird man die $\dot{r}_{\boldsymbol{\prime}} \approx \tilde{i} \alpha$ vergleichen dürfen, die, an Stelle unserer Glocken, auch sonst in Heiligthümern und beim Kult gebraucht wurden.

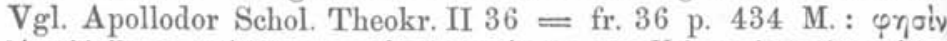

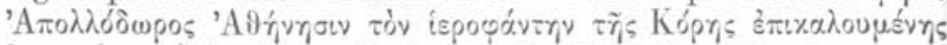

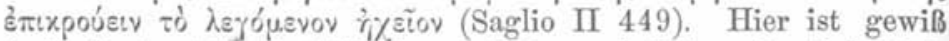
(s. Lobeck Aglaoph. 1225*) von keinen xúp3àov die Rede (wie Lenormant bei Saglio $575 \mathrm{f}$. wieder annimmt), sondern von einem Schlaginstrumente nach der Art des besprochenen ${ }^{8}$ ).

In diesen Zusammenhang möchte ich schlieBlich auch noch eine schwierige Petronstelle (c. 99) einrücken, die meines Wissens noch nicht endgiltig erledigt ist. Vor seiner Abreise hat sich Eumolpus mit Encolpius und Giton versöhnt; jetzt wollen sie gemeinsam zu Schiffe das Weite suchen. Ueber der Rührscene ist aber die festgesetzte Abfahrtszeit herangerïckt: adhuc loquebatur, cum crepuit ostium impulsum stetitque in limine barbis horrentibus nauta et 'moraris' inquit 'Eumolpe, tanquam propudium ignores'. Haud mora, omnes consurgimus etc. Man muß die verzweifelten Erklärungsversuche der früheren Interpreten bei Burmann nachlesen, um sich zu überzeugen, daß propudium nicht in Ordnung sein kann. Bald soll das Wort prope diem bedeuten, was sprachlich undenkbar ist, bald soll Tryphaena damit gemeint sein : 'nam propudia . . proprie meretrices' bemerkt Burmann. Die vorgeschlagenen Verbesserungen sind freilich eben so schlimm. Cuper empfahl prolubium $=$ 'promptum meum ad navigandum animum', Tornaesius properandum: das Eine matter als das Andre. Ich habe schon vor Jahren pro-

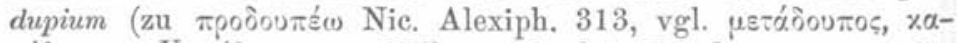

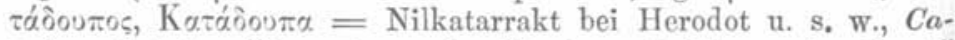
tadupa bei den Römern) an den Rand geschrieben; der Begriff 'Signal vor der Abfahrt' paßt genau an die strittige Stelle und würde durch die allerdings sonst nicht $\mathrm{zu}$ belegende Wortbil-

7) Freilich wenig urkundlich. Die Hauptbedenken gegen seine Auffassung bat schon Preller hervorgehoben.

$\left.{ }^{8}\right)$ Es braucht kaum erwähnt zu werden, daß die Alten auch die

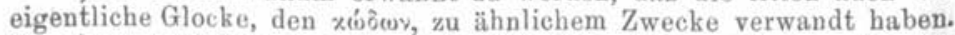
In einer Anekdote bei Strabo XIV 2, 21 p. 658 verlassen alle $\mathrm{Zu}$ -

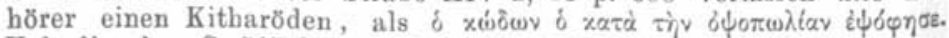
Vgl, die oben S. 515 behandelte Cicerostelle. 
dung aufs glücklichste ausgedrückt. Jetzt meine ich diese Vermuthung wenigstens frageweise vorlegen zu dürfen. Es ist wohl von einer Art Schiffsglocke die Rede, die wir uns etwa wie den Diskus bei Saglio construiert zu denken haben. ठov$\pi \varepsilon$ iv ist der richtige Terminus. Vgl. Xenoph. Anab. I 8, 18

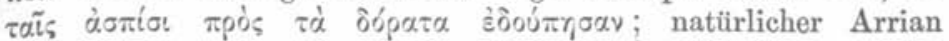

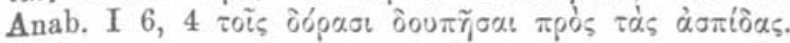

\section{Zur Einrichtung alter Kaufläden. (nach Herondas VII).}

Für die Veranschaulichung des Kleinhandels bei den Alten steht uns reiches Material zu Gebote, litterarisches wie archäologisches; um seine Verwerthung hat sich neuerdings besonders H. Blümner verdient gemacht. Vollkommen lebendig wird das jetzt durch den siebenten Mimus des Herondas, der uns bekanntlich in den Laden eines Schusters führt. Freilich ist er, vor Allem in der Anfangspartie, so lückenhaft und schwierig, daB man sich auch über die Scenerie und die Bühnenrequisiten, wenn ich so sagen darf, nicht auf den ersten Anlauf klar geworden ist. Einige Fragen der Art mögen hier behandelt werden, zum Theil im Anschluß an die Ausführungen Blümners in dieser Zeitschrift LI $134 \mathrm{ff}$.

Blümner nimmt an, daß die Begrïßung auf der Straße spiele vor der Thür von Kerdons Werkstatt, und daß Kerdon auch fertige Schuhwaare hinausbringen lasse. Erst in der Mitte, V. $55 \mathrm{ff}$. trete ein Scenenwechsel ein, indem Kerdon die Damen veranlasse, die Werkstatt selbst zu betreten. Ein solcher Scenenwechsel ist in diesen Dichtungen aber sonst nirgends nachweisbar, auch nicht im ersten oder vierten Stïck. Vollends unmöglich ist er in der Partie, wo Blümner ihn ansetzt, V. 55-83. Es findet sich hier nicht die leiseste Andeutung, die sich in jenem Sinne verwerthen

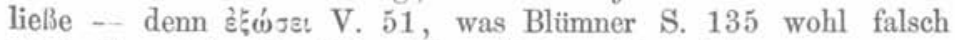
verstanden hat, bezieht sich auf das Herausholen aus einem Schranke. Von V. 54 bis V. 80 bleibt die Situation unver-

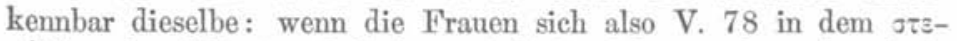

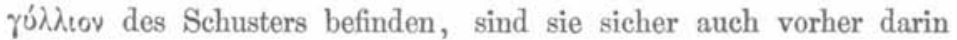
gewesen.

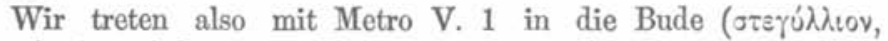
taberna) eines Schusters. Blümner sieht in den Räumen zugleich die Werkstatt. Dafür giebt es aber keinen Anhaltspunkt. Ein

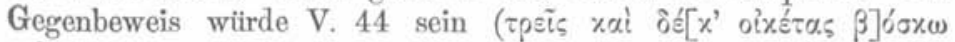
$x \tau \lambda$.), wenn hier nicht das ausschlaggebende Wort ergänzt wäre. Aber auch V. 4 f. können wir als Gegenbeweis verwerthen. Kerdon ruft einem Sklaven, Drimylos zu: 


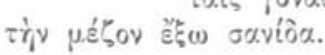

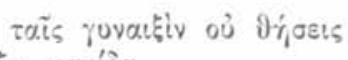

Hier fragt sich's zunächst, was wir unter der gavic zu verstehn haben. Bücheler, dem sich Dalmeyda, Meister u. A. anschliessen, versteht darunter eine tabula, ein Brett mit Waaren. Diese Deutung ist unmöglich, wie V. $12 \mathrm{f}$. ergiebt. Erst nachdem der säumige Drimylos die oaviç abgewischt hat, fordert Kerdon seine

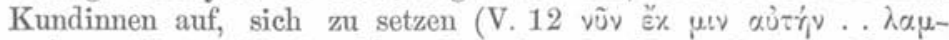

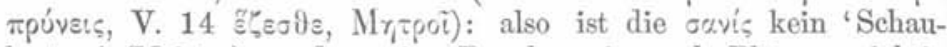
kasten' (Meister), sondern eine Bank, wie auch Blümner richtig angenommen hat. In der That sehn wir auch auf bildlichen Darstellungen des Schuhhandels die Käuferinnen sitzen, s. Th. Schreiber, kulturhistorischer Bilderatlas LXXII 7. LXXXVII 5.

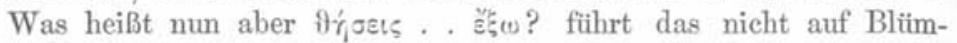
ners Auffassung zurück? Keineswegs; wir haben uns den Sklaven Drimylos mit einigen Genossen in einem mehr nach innen gelegenen Gelasse arbeitend vorzustellen; die oavi; ist eine von den drinnenstehenden Bänken, von denen Kerdon auch V. 40 f. nach einer sehr wahrscheinlichen Ergänzung zu sprechen scheint :

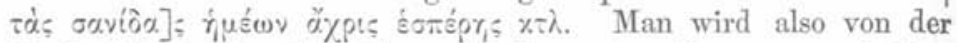
Werkstätte, in der die Sklaven arbeiten, einen Vorraum scheiden müssen, der als Kaufladen diente. Dieser Laden ist, ganz wie bei uns, direkt von der Straße her zugänglich, vgl. V. 122

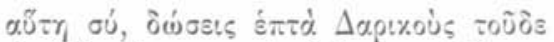

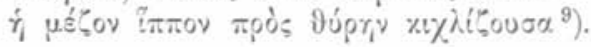

Seine Einrichtung ist sehr einfach. Sessel sind nicht vorhanden und eine Bank für einkaufende Damen wird aus einem Nebenraum herbeigeholt. Das wichtigste Ladengeräth sind die Wa arenschränke. Nachdem sich die Frauen gesetzt haben, befiehlt Kerdon dem Pistos, der offenbar ein bevorzugter Sklave ist und den Ladendiener macht:

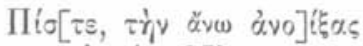

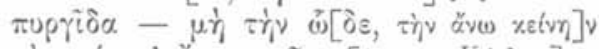

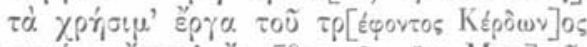

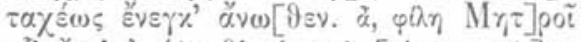

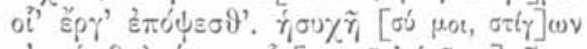

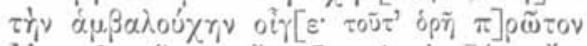

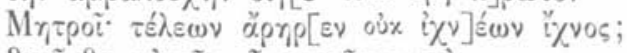

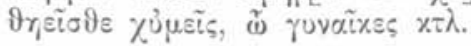

Soweit die Ergänzungen unsicher bleiben, sind sie durch keinen

9) Uebrigens sitzt auch Metriche im ersten Mimus in einem Raum, dessen Thür unmittelbar auf die Straße führte: nur so ist der Eingang verständlich. Vgl. Monceaux u. d. W. Domus bei D.-S. II S. $342^{6}$. 
Druck kenntlich gemacht. Ueber die Worte, die uns hier angehn, bleibt kein Zweifel ${ }^{10}$ ). Der Sklave soll eine $\pi \cup p \gamma$ is öffnen, um Waaren zu holen. Daß wir unter ropris einen Schrank, ein

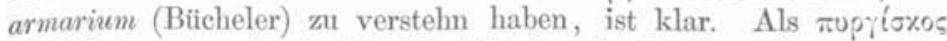
wird bei Aelian (v. h. IX 13, 10) das Geräth bezeichnet, hinter dem sich der ungeschlachte Dionysios von Heraklea vor seinen

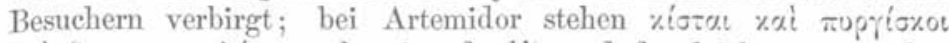

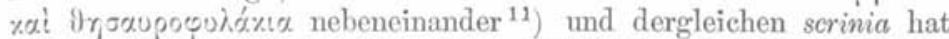
man auch bei Sextus Empirieus VII 102. IX 78 mit Recht

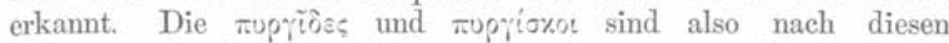
Belegen schmale Schränkchen, in denen man Kostbarkeiten aufhob. Hiernach werden sich auch auf den Kunstdenkmälern Beispiele nachweisen lassen. Auf einem Relief bei DarembergSaglio I Fig. 324 (= Fig. 852) steht ein Geräth mit geöffneten Thüren, das nach der Höhe des Davorsitzenden zu schließen etwa $1,25 \mathrm{~m}$. hoch und $0,40 \mathrm{~m}$. breit gedacht ist und oben mit einer als Pult zu benutzenden Platte abschlieBt; es wird durch Brettehen, auf denen Manuscripte und Geräthe liegen, in mehrere Fächer abgetheilt. Hier haben wir wohl einen

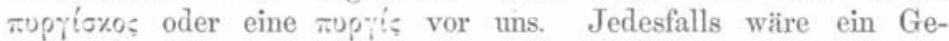
räth der Art für Kerdons Zwecke vorzüglich geeignet. Bei Kerdon hängen die Waaren nicht paarweise offen an der Wand, wie bei den Schustern auf den pompejanischen Forumsscenen. Seine šp; $\alpha$ liegen säuberlich in der $\sigma \alpha \mu \beta \alpha \lambda \circ \cup \chi_{0} \gamma_{i}$, die gleichfalls verschlieBbar ist (V. 19 f.). Bücheler, und mit ihm Meister, versteht darunter einen "Schuhkasten", d. h. einen Behälter mit mehreren Sandalen. Aber aus V. 51 s̃์ folgt doch wohl, daß die Schuhe einzeln hervorgeholt werden;

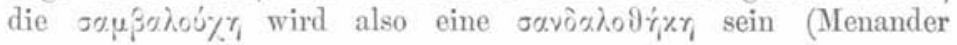

${ }^{10}$ ) Mein jetziger Text weicht freilich in Einzelheiten von meinem

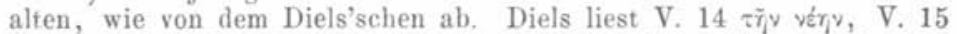

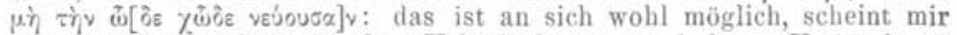
aber weniger Anhalt in dem Ueberlieferten zu haben. V. 17 hatte ich $\mu \tilde{\alpha}$ gesetzt. Aber $R$. Meister hat die feine und fruchtbare Beobachtung gemacht, daß Herondas $\mu \tilde{\alpha}$ nur Weibern in den Mund legt; auch seine Ansicht über den Grund dieser Erscheinung halte ich für zutreffend. Man muß daher $\tilde{\omega}$ oder nach V. 111 ¿े schreiben. Daß Kerdon V. 18 nicht Metro anredet, sondern eine andre Person, in diesem Zusammenhange also Pistos, ergiebt sich mit voller Sicherheit aus dem doppelten Mrizpot V. 17 und 20; der Dichter pflegt solche Anreden nicht umsonst zu setzen.

11) Meinekes Conjektur uppiøxot ist hier sehr überflüssig. Es ist bemerkenswerth, daß das seltne Wort in den Inschriften aus der Heimath des Artemidor wiederholt vorkommt, vgl. CIGr. III 4207. 4212. $4220^{\mathrm{b}}$ Add. $4340^{\mathrm{c}}$ Add. Es bezeichnet hier $\mu \nu \eta \mu \varepsilon i \alpha$, offenbar jene Grabthürme, über die Benndorf und Niemann, 'Reisen in Lykien' I 109 gehandelt haben. 
IV p. 165 M.), d. h. ein Kästchen für das einzelne Paar, was der Käufer mitbekam. Wir sind ja in einem eleganten Laden; der Meister zählt unendliche 'Novitäten' auf ( $\tau \dot{\alpha}$. vś $\alpha$ $\tau \alpha \tilde{u} \tau \alpha$. V. 56, wie nach einer richtigen Beobachtung Meisters statt $\gamma \varepsilon \dot{v} \varepsilon \alpha$ zu schreiben ist), und die Preise sind zum Theil

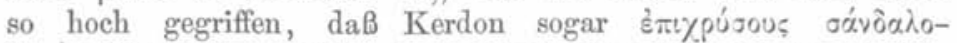
V̛́r $\alpha_{5}$ (Menander a. O.) mit in Kauf geben könnte. Diese Sandalenkästchen werden aus der $\pi \cup p \gamma i \zeta$ herausgeholt; sie stehen dort offenbar auf den Etagen-Brettchen, ganz wie in unsern Läden ${ }^{12}$ ).

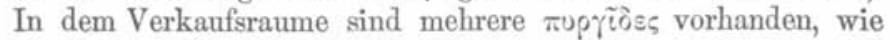
aus V. 14 hervorgeht; es fragt sich, in welcher Anordnung. Leider sind wir hier auf Vermuthungen angewiesen. Aus dem

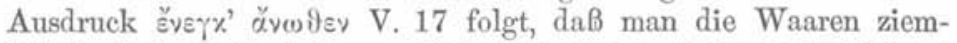
lich hoch herunter holen mußte; bei einem "Thurmschrank" ist das ja sehr begreiflich. Da aber von zwei tupriòs die Rede ist, wäre es wohl möglich, daß die eine auf die andre gestellt werden konnte, wie bei unsern Eckschränken. Auch das by-

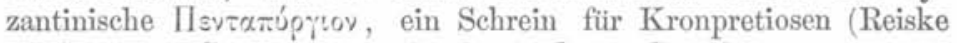
zu Constant. Cerim. p. 42 D 7), wird ein Geräth gewesen sein, das aus fünf $\pi$ opric\%ol bestand; man könnte annehmen, daB es sich in zwei Etagen, die untere von drei, die obere von zwei Schränkchen, gegliedert habe, wie jene mittelalterlichen Schreine, deren oberen Abtheilungen auch dureh den Zinnenkranz unverkennbar als Thürmchen charakterisiert sind. Doch reicht das Material, das mir bisher bekannt geworden ist, nicht aus, um hier eine feste und klare Anschauung zu gewinnen ${ }^{13}$ ).

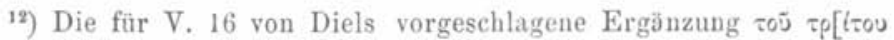

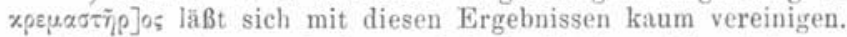

13) Bemerkenswerth ist es, daß der Taubenschlag bei den Römeru turris heißt (Varro de r. r. III 3, 6). Anch das beim Würfelspiel gebräuchliche Instrument mípyos (Agath. Anthol. Pal. IX 482, 23. Sidon. Ap. Ep. VIII 12) oder turricula (Mart. XIV 16 u. A.) ist kein Würfelbecher, wie immer wieder angenommen wird (z. B. von Friedländer zu Martial a. 0.), sonderu eiu wirkliches Thürmchen mit einem gewundenen oder treppenartigen Gange, durch den die Knöehel heruntersprangen, wie bei unserm Tivolispiel. Vgl. Sidon, a. 0. tessera fre-

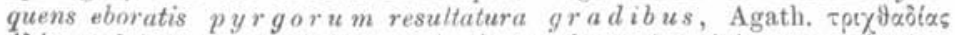

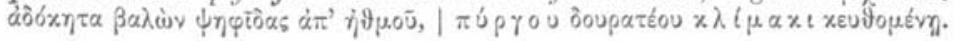

Tübingen.

o. Crusius. 